\title{
La covariación en las tareas de los libros universitarios de precálculo: el caso de las razones trigonométricas
}

\author{
Covariation in Precalculus Textbooks Tasks: \\ a Case of Trigonometric Ratios
}

\author{
Jhony Alexander Villa-Ochoa* \\ ORCID iD 0000-0003-2950-1362 \\ Ferney Tavera Acevedo** \\ ORCID iD 0000-0002-9998-9204
}

\begin{abstract}
Resumen
La inclusión del estudio de la variación en la cotidianidad escolar es un propósito en varios currículos alrededor del mundo. Sin embargo, no es clara la manera en que los libros de texto universitarios llevan a cabo este propósito. Por lo tanto, se realizó un análisis de las tareas de libro de texto con el fin de identificar la manera en que la covariación está presente en tales tareas. Se usaron herramientas de análisis de contenido para analizar las tareas de cinco libros universitarios de precálculo. Los resultados de este análisis muestran un énfasis especial en tareas que desaprovechan oportunidades para matematizar la covariación. Se ofrece una tipología de tareas y se sugiere la posibilidad de reorganizar algunas de ellas con el fin de promover el estudio de la covariación.
\end{abstract}

Palabras clave: Covariación. Libros de Texto de Precálculo. Razones Trigonométricas. Tipos de Tareas.

\begin{abstract}
Variation learning has been recommended in several academic curricula around the world; however, the manner in which university textbooks carry out this purpose is unclear. We carried out an analysis of the textbook tasks in order to identify the way in which covariation is present in the trigonometry tasks. Content analysis tools were used to analyze the tasks of five university precalculus textbooks. The study shows a special emphasis on tasks that miss opportunities to mathematize the covariation. This article offers a typology of tasks and also suggests the possibility of reorganizing some of them, in order to promote covariation studies.
\end{abstract}

Keywords: Covariation. Precalculus Textbooks. Trigonometric Ratios. Task Types.

\section{Introducción}

En las últimas décadas, el estudio de la variación y su inclusión en las matemáticas escolares ha ocupado las agendas de varios investigadores (THOMPSON, 1994; CARLSON

\footnotetext{
* Doctor en Educación en el área de Educación Matemática de la Universidad de Antioquia (UdeA). Profesor Titular de la Universidad de Antioquia (UdeA), Medellín, Colombia. Dirección postal: Calle 67 No. 53 - 108, bl 9-120, Ciudad Universitaria, Medellín, Colombia, Código postal 050010. E-mail: jhony.villa@udea.edu.co.

** Magíster en Educación Matemática de la Universidad de Medellín (UdeM). Profesor de la Escuela Normal Superior Genoveva Díaz, San Jerónimo, Antioquia, Colombia. Dirección postal: Calle 67 No. 53 - 108, Ciudad Universitaria, Medellín, Colombia, Código postal 050010. E-mail: ftavera827@yahoo.es.
} 
et al., 2002; VASCO, 2006; TALL, 2009; VILLA-OCHOA, 2012; VILLA-OCHOA, GONZALEZ-GÓMEZ, CARMONA-MESA, 2018, FIALLO-LEAL, PARADA-RICO, 2018; BARAJAS-ARENAS, PARADA-RICO, MOLINA ZABALETA, 2018). La mayoría de los investigadores coincide en la necesidad de ofrecer oportunidades en el aula que permitan a los estudiantes experimentar, analizar e interpretar situaciones en las que la noción de variable y de variación tenga un papel primordial.

Tall (2009), por ejemplo, considera que el corazón del análisis matemático está en la variación y sugiere que, para abordar las temáticas del cálculo, es indispensable hacer énfasis en los procesos funcionales y dinámicos que subyacen en cada uno de los conceptos allí tratados. Carlson et al. (2002) formularon un marco conceptual que sirvió para conectar la noción de covariación con ideas del cálculo. En su estudio, estos investigadores observaron que en la literatura internacional se documentan cada vez más las dificultades que tienen los estudiantes en la comprensión de los conceptos de función, límite, derivada e integral. Para estos autores, estas dificultades se asocian a habilidades de razonamiento covariacional empobrecidas.

Carlson et al. (2002) encontraron que los estudiantes universitarios que participaron de su investigación no lograron modelar relaciones funcionales que involucraban la razón de cambio de una variable cuando esta varía continuamente con otra variable, porque no coordinaron la razón de cambio promedio con cambios fijos en la variable independiente para el dominio de una función, ni tampoco coordinaron la razón de cambio instantánea con cambios continuos en la variable independiente.

En Latinoamérica existe una línea de investigación denominada Pensamiento y Lenguaje Variacional. Esta línea se encarga de estudiar los fenómenos que suceden con la enseñanza, el aprendizaje y la comunicación de saberes matemáticos propios de la variación y el cambio, tanto en el sistema educativo como en el medio social donde se desempeñan los estudiantes y/o profesores (CANTORAL, 2004).

Para Cantoral (2004), estudiar la variación implica centrarse en las causas que originan un fenómeno, de tal manera que a través de la variación se cuantifique el cambio por medio del análisis y la interpretación de lo que está cambiando, cómo cambia y cuánto cambia; luego se da paso a los procesos algebraicos y analíticos. Las prácticas propias de la variación son las encargadas de dotar de sentido y significado a los conceptos matemáticos abordados en cada clase y, por ende, a los procedimientos que allí se asocian (CABALLERO-PEREZ; CANTORAL, 2013).

El estudio de la matemática a través de un enfoque covariacional también está presente 
en el estudio de conceptos previos al cálculo, por ejemplo, el estudio de funciones exponenciales (FERRARI-ESCOLÁ; MARTÍNEZ-SIERRA; MÉNDEZ-GUEVARA, 2016); cuadráticas (VILLA-OCHOA, 2012) y trigonométricas (MOORE, 2014). Para Moore (2014), la conexión entre la medida del ángulo y la medida de los arcos, así como una concepción de que el radio puede ser una unidad de medida contribuyen a generar significados trigonométricos que abarcan los contextos no solo de la trigonometría del círculo unitario sino también del triángulo rectángulo.

Diversos estudios han señalado que, aunque en la trigonometría se utilizan conceptos propios de la variación (variables, razón, proporción, función), no siempre se pone de relieve la comprensión de relaciones dinámicas de covariación (MONTIEL; JÁCOME, 2014; TAVERA; VILLA-OCHOA, 2015); por tanto, se requiere de una didáctica especial y de un cambio en el lenguaje que permita una comprensión dinámica de la función (MONTIEL; BUENDÍA-ÁBALOS, 2013). En este tipo de necesidades los problemas, recursos y discursos presentes en los libros de texto cumple un importante papel (MONTIEL; JÁCOME, 2014; TAVERA; VILLA-OCHOA, 2015).

La preocupación por el rol que cumplen los libros de texto en la comprensión de la trigonometría se fundamenta en estudios recientes que informan la relevancia que tiene este recurso, pues tiene el potencial de dar forma a la manera en que se enseña y aprende matemáticas (SELVA; BORBA, 2014; SON; DILETTI, 2017). Para algunos profesores, el libro de texto es el principal referente de trabajo, en buena parte, por la ausencia de otros materiales que los orienten en relación con lo que debe enseñarse y cómo debe hacerse (SELVA; BORBA, 2014).

Para Randahl (2012), los libros de texto no solo se utilizan como guía para explicar los conceptos matemáticos, también sirven como objeto de estudio, material de consulta o fuente bibliográfica que recopila ejercicios y problemas que se plantean para que los estudiantes se preparen y evidencien el aprendizaje en determinado momento. Investigaciones han evidenciado que la organización del contenido, los problemas y las maneras en que el profesor y los estudiantes interactúan con los libros de texto, ofrecen oportunidades para mejorar el desempeño académico de los estudiantes (SON; DILETTI, 2017), pero también van más allá de las matemáticas, buscando la normalización de la conducta de los estudiantes (SILVA et al., 2018).

Así, los libros de texto no son un recurso neutro en los procesos de producción de conocimiento matemático escolar; más allá de ello, son importantes porque actúan como una herramienta ideológica que se construye en consenso, pensando en los beneficios que recibiría 
la comunidad educativa que decida emplearlos, dado que es allí donde se evidencia la aplicabilidad de un currículo ya establecido. Para Cantoral, Montiel y Reyes-Gasperini (2015), el análisis de un libro de texto es fundamental para la investigación educativa en la medida en que brinda visiones institucionalizadas del conocimiento matemático que, con frecuencia, suelen ser distantes de los estudiantes.

El estudio de la covariación en los libros de texto requiere de la atención a los contextos, conceptos, procesos y procedimientos que involucran fenómenos de cambio. Estos aspectos se interrelacionan en las situaciones, problemas o tareas que se proponen allí. En coherencia con ello, y con base en la noción de covariación, este artículo se propone dar una respuesta a la pregunta ¿Cuáles tipos de tareas pueden identificarse sobre las razones trigonométricas en libros de texto de precálculo?

\section{Antecedentes teóricos}

En Colombia se ha propuesto promover el desarrollo del pensamiento variacional a lo largo de los distintos niveles de escolaridad. Por ejemplo, para la Educación Básica y Media, el Ministerio de Educación Nacional (MEN) sugiere que los currículos propendan por "el reconocimiento, la percepción, la identificación y la caracterización de la variación y el cambio en diferentes contextos, así como su descripción, modelación y representación en distintos sistemas o registros simbólicos, ya sean verbales, icónicos, gráficos o algebraicos" (COLOMBIA-MEN, 2006, p. 66).

Se espera que los estudiantes puedan explorar, reflexionar, deducir, conjeturar y plantear nuevas situaciones frente a las relaciones funcionales y dinámicas que se generan entre los distintos conceptos matemáticos (TAVERA; VILLA-OCHOA, 2015). Bajo esta consideración, el estudio de la variación puede considerarse como un enfoque para las matemáticas escolares.

En la literatura internacional se han desarrollado perspectivas y constructos teóricos particulares, entre ellos, se reconocen: el razonamiento covariacional (CARLSON et al., 2002; THOMPSON; CARLSON, 2018), el pensamiento y lenguaje variacional (CANTORAL, 2004; CABALLERO-PEREZ; CANTORAL, 2013) y el pensamiento funcional (STEPHENS et al., 2017; PINTO; CAÑADAS, 2018).

El estudio de la variación en los cursos de cálculo tiene un creciente interés en Latinoamerica (FIALLO-LEAL; PARADA-RICO, 2014, 2018; RUEDA; PARADA, 2016; PARADA; CONDE; FIALLO, 2016; CABEZAS; MENDOZA, 2016; ARANDA; CALLEJO, 
2017; FLORES; GARCÍA-GARCÍA， 2017; ANTONIO-ZAMBRANO; ESCUDEROÁVILA; FLORES-MEDRANO, 2019). Parada, Conde y Fiallo (2016, p. 1033) proponen que comprender la variación implica "explicar cómo se relacionan las magnitudes variables en un problema particular, así como medir y analizar cómo cambian estas magnitudes”. Para estos investigadores, el cambio puede considerarse como un núcleo conceptual del cálculo, el cual tiene su génesis en la solución de problemas "en los que se requiere identificar y usar variables, no como una letra que representa un número o el valor desconocido en una ecuación, sino las variables como cantidades mesurables, que cambian cuando las situaciones en que ocurren cambian" (PARADA; CONDE; FIALLO, 2016, p. 1035).

Además de la identificación de la correlación y de la manera en que cambian las magnitudes, otros trabajos han puesto de relieve la noción de imagen de covariacion y de coordinación de estas imágenes (CARLSON et al., 2002; SALDANHA; THOMPSON, 1998). Así, para Saldanha y Thompson (1998), una comprensión de la covariación involucra la actividad de sostener en la mente, de manera simultánea, una imagen de dos valores de cantidades o magnitudes; para los autores, esto involucra la coordinación de las dos cantidades, esto es, reconocer la dependencia entre ellas, pues para cada valor de una cantidad se debe dar cuenta de que a la otra cantidad también le corresponde un valor en cada instante.

Estas nociones fueron la base para que Carlson et al. (2002) definieran el razonamiento covariacional como las actividades cognitivas que se involucran en la coordinación de dos cantidades que varían mientras se atiende a las formas en que cada una de ellas cambia con respecto a la otra. Para los autores, este tipo de razonamiento va desde la coordinación de los valores entre dos variables hasta una comprensión de la tasa de variación instantánea.

Con base en las anteriores consideraciones, la variación como un enfoque para las matemáticas escolares sugiere trascender las interpretaciones clásicas del álgebra, la aritmética o la geometría para posicionarse en una interpretación dinámica de los diferentes objetos matemáticos. Todo ello hace que el reconocimiento y cuantificación de cambios de estado, la interpretación dinámica de las variables, la coordinación de cambios entre variables y la noción de razón, la instanciación a través de una figura se conviertan en oportunidades para introducir posteriores ideas formales de la función y otros temas del análisis matemático.

\section{Metodología}

Conforme se mencionó antes, existen investigaciones que documentan que los libros 
de texto tienen el potencial de dar forma a la manera en que se enseña y aprende matemáticas (SON; DILETTI, 2017). En este sentido, se esperaría que los libros de texto sean recursos que provean oportunidades para que profesores y estudiantes se involucren en ambientes que permitan estudiar la variación. Para dar cuenta de las oportunidades que ofrecen los libros de texto para el estudio variacional de la trigonometría se desarrolló una investigación cualitativa en la que se usaron herramientas del análisis de contenido.

El análisis de contenido se considera como una "técnica que pretende dilucidar la naturaleza del discurso generado en una realidad social, la cual está determinada a través de la producción documental sustentada en los libros de texto" (PINO; BLANCO, 2008, p. 73). Con base en esta descripción, es posible colegir que este método intenta generar razonamientos discursivos o inferencias a partir de los datos registrados en cualquier tipo de lenguaje que exprese comunicación (por ejemplo: verbal, escrito, pictográfico etc.); a causa de ello, es posible analizar e interpretar la manera en que los libros de texto presentan las tareas propuestas.

En esta investigación se analizaron las tareas que cinco libros de texto universitarios propopen para el estudio de las relaciones trigonométricas en un enfoque de la variación para las matemáticas escolares.

\subsection{Los datos y su análisis}

Para obtener un acercamiento a la pregunta de investigación se buscaron libros de texto significativos en el área Metropolitana de Medellín (Colombia). Por tal motivo, se determinaron los siguientes criterios: (i) que sean textos recomendados por los departamentos de Matemáticas o Ciencias Básicas en universidades acreditadas en la Ciudad, (ii) que sean utilizados por los estudiantes universitarios en los primeros años de educación superior y, finalmente, (iii) que desarrollen las temáticas propias de la trigonometría. En particular, este artículo se fundamenta en la trigonometría del triángulo, pues en la literatura internacional se reporta un distanciamiento entre esta trigonometría y la analítica; en esta última, el concepto de función trigonométrica cobra sentido (MONTIEL; BUENDÍA-ÁBALOS, 2013; MOORE, 2014).

En el Cuadro 1 se presenta la lista de libros de texto universitarios que se seleccionaron para analizar las tareas propuestas sobre el estudio de las relaciones trigonométricas en una perspectiva variacional. En la lista, dos de los libros son producciones propias de las universidades y los otros tres son traducciones al español de libros de 
editoriales internacionales.

\begin{tabular}{|c|c|c|c|c|}
\hline Autor(es) & Año & Título del libro de texto & Edición & Editorial \\
\hline Buriticá*, B. & 2012 & Álgebra y Trigonometría & Tercera & $\begin{array}{l}\text { U de A. (Programa U de } \\
\text { (a) }\end{array}$ \\
\hline $\begin{array}{l}\text { Stewart, J; Redlin, L; } \\
\text { Watson, S }\end{array}$ & 2012 & $\begin{array}{l}\text { Precálculo. Matemáticas para el } \\
\text { Cálculo }\end{array}$ & Sexta & Cengage Learning \\
\hline $\begin{array}{l}\text { Swokowski, E. W; Cole, } \\
\text { J. A }\end{array}$ & 2009 & $\begin{array}{l}\text { Álgebra y Trigonometría con } \\
\text { Geometría Analítica }\end{array}$ & $\begin{array}{l}\text { Décimo } \\
\text { segunda }\end{array}$ & Cengage Learning \\
\hline Sullivan, M & 2007 & Álgebra y Trigonometría & Séptima & Pearson \\
\hline $\begin{array}{l}\text { Mejia*, F; Álvarez, R; } \\
\text { Fernández, H. }\end{array}$ & 2005 & Matematicas previas al cálculo & Primera & $\begin{array}{l}\text { Sello Editorial Universidad } \\
\text { de Medellín }\end{array}$ \\
\hline
\end{tabular}

\section{Cuadro 1 - Lista de libros de texto universitarios} Fuente: elaborado por los autores

En un reciente survey, Son y Diletti (2017) clasificaron en dos amplias categorías los intereses de investigación sobre libros de texto; a saber, (i) estudios que centran los análisis en el desarrollo de un contenido temático y (ii) estudios que se centran en el análisis de los problemas. En relacion con la segunda categoría, los autores encontraron que las características del contexto y del problema, los tipos de respuesta, las demandas y expectativas cognitivas, profundidad del conocimiento y la relevancia de elementos no textuales fueron los aspectos que más se han estudiado en la investigación.

Para este estudio, el análisis se centró en las tareas propuestas por los libros de texto en las unidades temáticas de trigonometría del triángulo. Esta decisión se fundamentó en los trabajos de Carlson et al. (2002) y Villa-Ochoa (2012), para quienes el razonamiento covariacional de los estudiantes puede evidenciarse en el desarrollo de tareas específicas.

Si bien la comprensión de la covariación en los estudiantes sugiere la actividad de sostener en la mente la coordinación del cambio de dos o más cantidades (dependencia), en los libros de texto se propone identificar las oportunidades que se ofrece para el reconocimiento de la dependencia entre dos o más cantidades variables que constituyen la actividad de resolver la tarea.

Con base en lo anterior, todas las tareas se resolvieron de manera individual, por parte de cada investigador, con el fin de caracterizar la coordinación entre las cantidades (variables) que intervienen en ellas; en otras palabras, identificar la manera en que se promueve o no el reconocimiento de cantidades (variables y estáticas), las relaciones entre ellas y su interpretación como covariación. De forma posterior, los investigadores se reunieron y discutieron sus hallazgos y elaboraron un conjunto de dos categorías con su respectiva 
tipología de tareas.

\section{Resultados}

Los resultados de este estudio muestran que las tareas propuestas por los libros de texto son, principalmente, tareas que pueden resolverse con una interpretación estática de la relación entre las cantidades; es decir, tareas en las que las cantidades que intervienen no representan variables, sino valores únicos desconocidos. Solo en tres de los cinco libros de texto se pueden reconocer tareas que requieren de una interpretación dinámica; es decir, tareas en las que las cantidades son variables y la relacion entre cantidades exigen una interpretación de una función. A pesar de ello, la cantidad de estas tareas es mínima comparada con la cantidad en la categoría de tareas estáticas. En la Tabla 1 se presentan la frecuencia de estas tareas en cada categoría y tipología.

Tabla 1 - Frecuencia de tareas por tipología en los libros de texto

\begin{tabular}{|c|c|c|c|c|c|}
\hline \multirow[t]{2}{*}{ Título del libro de texto } & \multicolumn{3}{|c|}{ Tareas con interpretación estática } & \multicolumn{2}{|c|}{ Tareas dinámicas } \\
\hline & $\begin{array}{l}\text { Aplicación } \\
\text { directa }\end{array}$ & $\begin{array}{l}\text { Composición } \\
\text { de razones }\end{array}$ & $\begin{array}{l}\text { Seudo- } \\
\text { dinámicas }\end{array}$ & $\begin{array}{l}\text { Comparación } \\
\text { de estados }\end{array}$ & Covariación \\
\hline $\begin{array}{l}\text { Álgebra } \\
\text { trigonometría }\end{array}$ & 21 & 24 & 0 & 0 & 0 \\
\hline $\begin{array}{l}\text { Precálculo. } \\
\text { Matemáticas para el } \\
\text { cálculo }\end{array}$ & 63 & 108 & 2 & 4 & 0 \\
\hline $\begin{array}{lr}\text { Álgebra } & \text { y } \\
\text { trigonometría } & \text { con } \\
\text { geometría analítica } & \end{array}$ & 33 & 105 & 2 & 0 & 0 \\
\hline $\begin{array}{l}\text { Álgebra } \\
\text { trigonometría }\end{array}$ & 24 & 90 & 4 & 1 & 0 \\
\hline $\begin{array}{l}\text { Matemáticas previas al } \\
\text { cálculo }\end{array}$ & 12 & 60 & 1 & 1 & 0 \\
\hline $\begin{array}{c}\text { Total } \\
\end{array}$ & 153 & 387 & 9 & 6 & $\mathbf{0}$ \\
\hline
\end{tabular}

Fuente: elaborado por los autores

En la Tabla 1 se enuncia un conjunto de tipologías para cada categoría. A continuación, se ilustra cada una de ellas.

\subsection{Tareas con interpretación estática de las razones trigonométricas}

En esta categoría se agruparon aquellas tareas en las que no se requiere una interpretación de la correlación como una covariación entre las cantidades. Este tipo de contextos privilegia el cálculo de cantidades que, generalmente, se asocian a incógnitas (valores fijos aunque desconocidos) y no a variables ni a funciones. En los libros de texto que 
se analizaron se encontraron tres tipologías de tareas. Cada una de ellas obedece al uso de contenido matemático que se exige en la solución de la tarea. A continuación se describen y ejemplifican estas tipologías.

\subsubsection{Tareas por aplicación directa de una razón (estática)}

Para solucionar tareas asociadas a esta tipología, los estudiantes deben reconocer primero cuál es la razón trigonométrica (es decir: seno, coseno, tangente, cotangente, secante, cosecante) que se inscribe en la solución de la tarea; una vez que identifican esta razón, el resto de la solución involucra la aplicación de algunas expresiones algebraicas. Puede representarse esta tipología de tareas bajo la siguiente expresión: $\operatorname{raz}(\alpha)=\frac{a}{b}$.

En esta expresión, la raíz raz representa a cualquiera de las seis razones trigonométricas y los símbolos $a, b$ hacen alusión a las dos cantidades (no variables), de las cuales una de ellas es la incógnita y la otra es el valor numérico conocido (dado) que permite resolver satisfactoria y de manera inmediata la tarea planteada.

A continuación, en la Figura 1, se presenta uno de los enunciados extraído de Buriticá (2012, p. 223) con el cual se ejemplifica esta tipología:

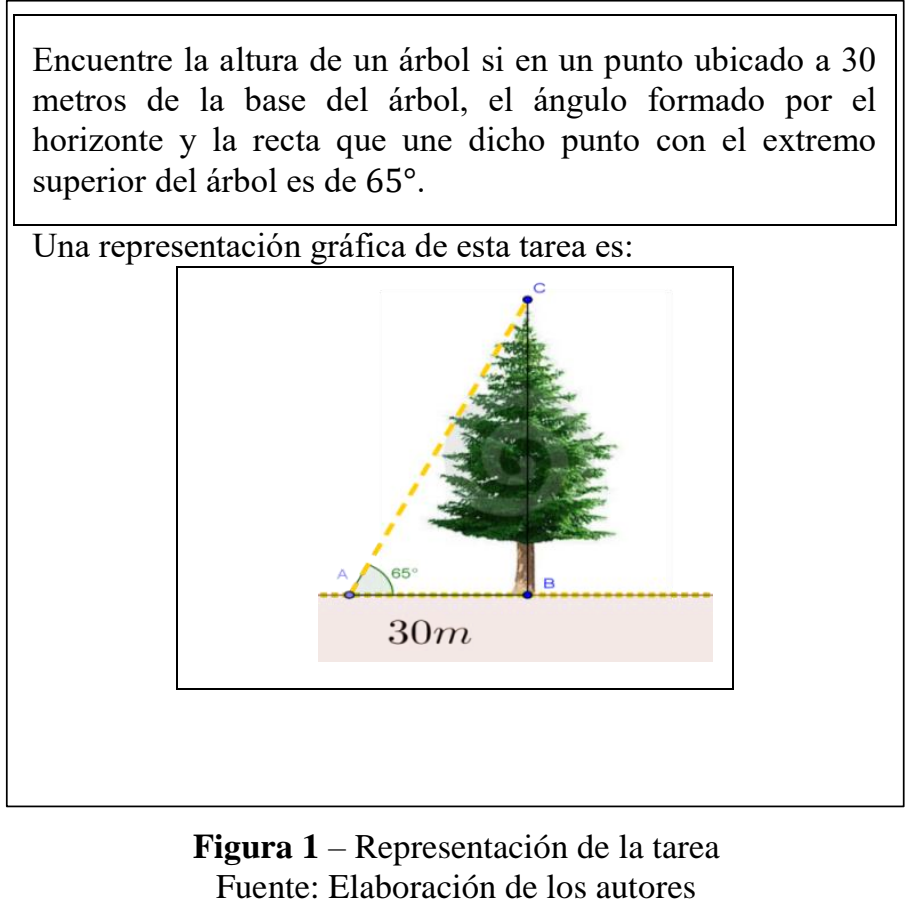

En la Figura 1, $\overline{\mathrm{AB}}$ representa la distancia que hay entre la base del árbol y el punto mencionado $\mathrm{A}$ (dato conocido); $\overline{\mathrm{BC}}$ representa la altura del árbol (incógnita) y $\overline{\mathrm{AC}}$ representa la línea visual del punto A y la parte superior del árbol (incógnita). 
Este enunciado obedece a una expresión algebraica de la siguiente manera:

$$
\tan 65^{\circ}=\frac{\mathrm{BC}}{30}
$$

Tanto en el enunciado como en la expresión algebraica que lo representa es posible observar la presencia de cantidades fijas conocidas (constantes como: ángulo de elevación y la distancia del punto mencionado al pie del árbol) y una desconocida solicitada (la altura del árbol). Esta tipología de tareas ofrece oportunidades para que los estudiantes asocien una relación trigonométrica con los datos presentados en el enunciado y que, a su vez, utilicen técnicas algebraicas para encontrar un resultado. Sin embargo, como se discutirá más adelante, este tipo de tareas no trasciende hacia una interpretación de las razones trigonométricas como representaciones de covariación entre cantidades; ni de las cantidades que intervienen en ellas (ángulos y medidas de los segmentos del triángulo) como variables.

\subsubsection{Tareas por composición de razones (estáticas)}

Esta tipología de tareas requiere de la aplicación de dos o más razones trigonométricas (no consideradas como manifestación de una covariación) para calcular los valores solicitados. Estos obedecen a la siguiente forma:

$$
\operatorname{raz}_{1}\left(\alpha_{1}\right)=\frac{a_{1}}{b_{1}}, \quad \operatorname{raz}_{2}\left(\alpha_{2}\right)=\frac{a_{2}}{b_{2}}, \quad \ldots \quad \operatorname{raz}_{\mathrm{n}}\left(\alpha_{\mathrm{n}}\right)=\frac{\mathrm{a}_{\mathrm{n}}}{\mathrm{b}_{\mathrm{n}}}
$$

En estas expresiones $r a z_{1}, r a z_{2}, \ldots, r a z_{n}$ representan a cualquiera de las seis razones trigonométricas y los símbolos $a_{1}, a_{2}, \ldots a_{n}$ y $b_{1}, b_{2} \ldots b_{n}$ representan cantidades (generalmente no entendidas como variables) donde algunas de ellas son las incógnitas y las otras son valores numéricos dados. En la resolución de la tarea se presentan, adicionalmente, algunas condiciones que permiten relacionar las $a_{i}$ con alguna otra cantidad (sea de la forma $a_{j}$ o $b_{k}$ ), de tal manera que posibilite resolver la tarea que se plantea. La expresión $r a z_{n}$ también puede referirse a la necesidad de utilizar la ley del seno o ley del coseno para poder resolver los cuestionamientos de la tarea propuesta.

A continuación, en la Figura 2, se presenta uno de los enunciados extraído de Mejía, Álvarez e Fernández (2005, p. 486). 


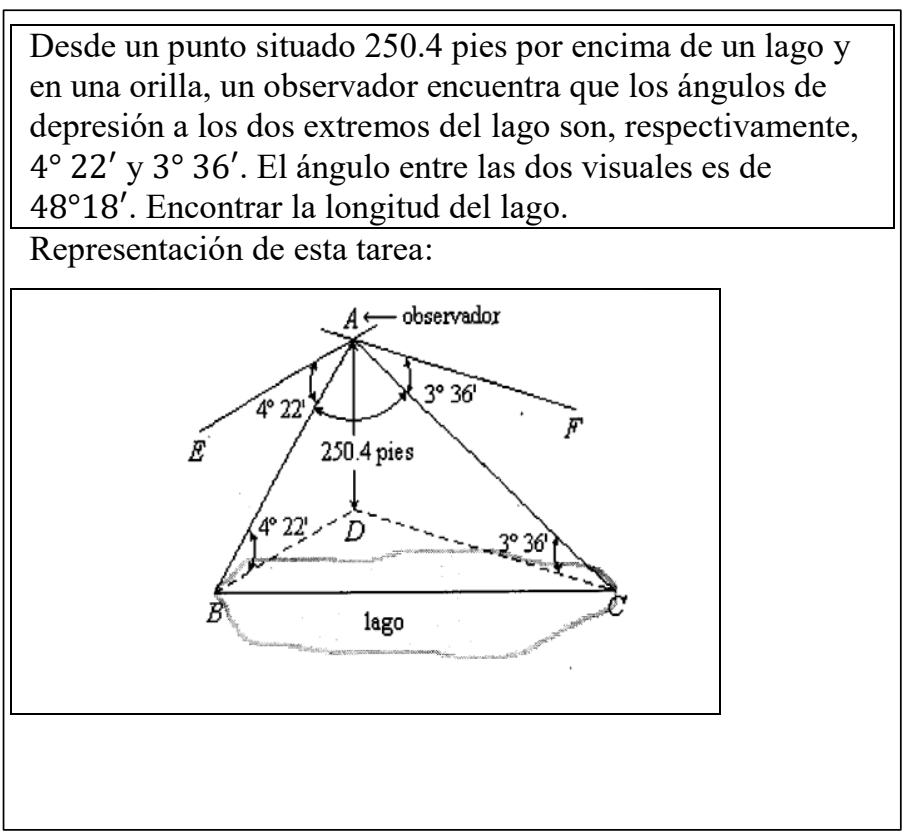

Figura 2 - Representación gráfica de la tarea

Fuente: Mejía, Álvarez y Fernández (2005, p. 486)

Según el planteamiento de esta tarea, se tiene que el ángulo BAC mide $48^{\circ} 18^{\prime} ; \overline{\mathrm{AD}}$ representa la altura que hay entre el observador y un punto situado D (este segmento también es perpendicular a $\overline{\mathrm{BD}}$ y a $\overline{\mathrm{DC}}$ ); $\overline{\mathrm{BC}}$ representa la longitud del lago; $\overline{\mathrm{AE}}$ es paralelo $\overline{\mathrm{BD}}$ y, de igual forma, $\overline{\mathrm{AF}}$ es paralelo $\overline{\mathrm{CD}}$.

Este enunciado obedece a las siguientes expresiones algebraicas:

$$
\begin{aligned}
& \sin 4^{\circ} 22^{\prime}=\frac{250.4}{\mathrm{AB}} \\
& \sin 3^{\circ} 36^{\prime}=\frac{250.4}{\mathrm{AC}}
\end{aligned}
$$

Utilizando la notación anterior, se tiene que $\mathrm{b}_{1} \mathrm{y}_{2}$ son las medidas respectivas de $\overline{\mathrm{AB}}$ y $\overline{\mathrm{AC}}$. Asimismo, se tiene que las relaciones entre las longitudes de estos dos segmentos se obtienen a través de la denominada ley del coseno. Así:

$$
\mathrm{BC}^{2}=\mathrm{AB}^{2}+\mathrm{AC}^{2}-2(\mathrm{AB} \times \mathrm{AC}) \cos 48^{\circ} 18^{\prime}
$$

Al igual que en el ejemplo anterior, se observa la presencia de cantidades fijas conocidas que son constantes (v.g. ángulos de elevación y de depresión, altura que hay entre el observador y un punto situado), y otras desconocidas (v.g. longitud del lago, distancia que hay entre el observador y los dos extremos del lago, distancia que hay entre el punto situado y los dos extremos del lago). Este tipo de tarea permite apreciar la capacidad del estudiante para asociar una relación trigonométrica con los datos presentados en el enunciado y, a su vez, utilizar técnicas algebraicas para encontrar un resultado; sin embargo, este tipo de tareas 
tampoco trascienden hacia una interpretación de las razones trigonométricas como funciones, ni a las cantidades que intervienen en ellas como variables (ángulos y medidas de los segmentos del triángulo).

\subsubsection{Tareas con interpretación de las razones trigonométricas seudo-dinámicas}

Esta tipología de tareas se fundamenta en la presentación explícita o implícita de cantidades variables, las cuales producen algún tipo de cambio por ser aparentemente dinámicas. Sin embargo, los movimientos allí generados se limitan a reducir la tarea a preguntas por un valor numérico particular que satisface el enunciado.

A continuación, en la Figura 3, se presenta uno de los enunciados extraído de Mejía et al. (2005, p. 488) para ejemplificar esta tipología de tareas:

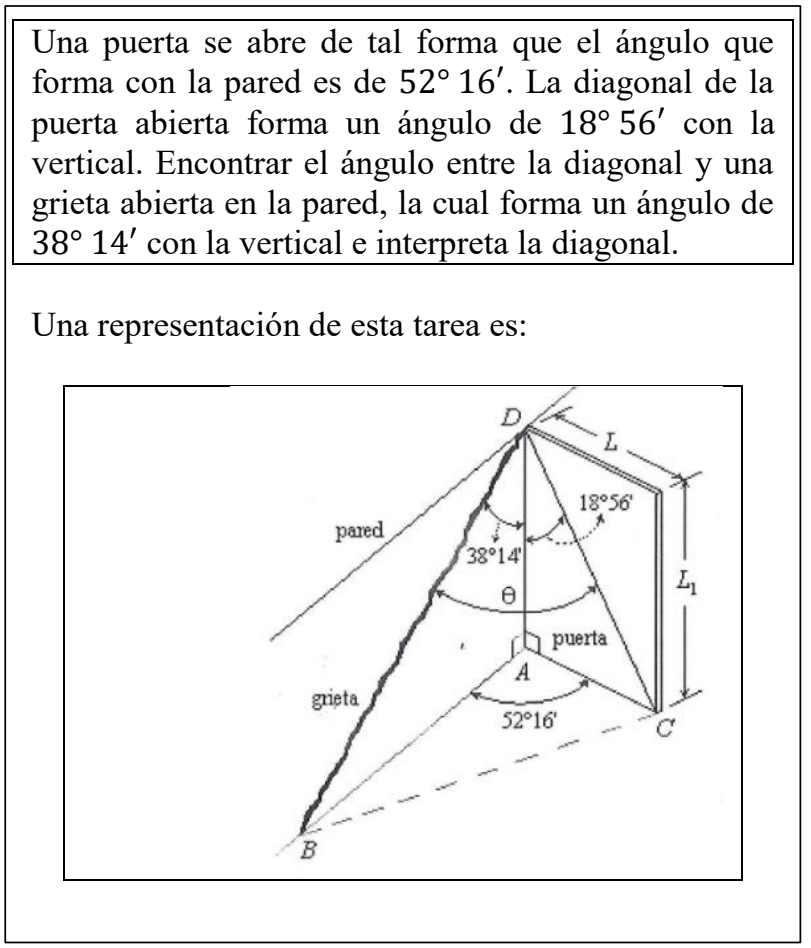

Figura 3 - Representación de la tarea

Fuente: Mejía, Álvarez y Fernández (2005, p. 489)

Según lo planteado en esta tarea, se tiene que el ángulo BAC mide $52^{\circ} 16^{\prime}$; el ángulo $\mathrm{ADC}$ mide $18^{\circ} 56^{\prime}$; y el ángulo $\mathrm{BDA}$ mide $38^{\circ} 14^{\prime} ; \overline{\mathrm{AD}}$ representa la altura de la puerta; $\overline{\mathrm{AC}}$ representa el ancho de la puerta; $\overline{\mathrm{DC}}$ representa la diagonal de la puerta; $\overline{\mathrm{DB}}$ representa la distancia que hay entre la parte superior izquierda de la puerta y un punto situado llamado B; $\overline{\mathrm{BC}}$ representa la distancia que hay entre el punto situado $\mathrm{B}$ y la parte inferior derecha de la puerta; $\overline{\mathrm{BA}}$ representa la distancia entre el punto situado $\mathrm{B}$ y la parte inferior izquierda de la puerta. 
Del enunciado se pueden extraer información para las siguientes expresiones algebraicas:

$$
\begin{aligned}
& \cos 18^{\circ} 56^{\prime}=\frac{\mathrm{AD}}{\mathrm{DC}}=\frac{\mathrm{L}_{1}}{\mathrm{DC}} \\
& \cos 38^{\circ} 14^{\prime}=\frac{\mathrm{AD}}{\mathrm{BD}}=\frac{\mathrm{L}_{1}}{\mathrm{BD}} \\
& \tan 18^{\circ} 56^{\prime}=\frac{\mathrm{AC}}{\mathrm{L}_{1}} \\
& \tan 38^{\circ} 14^{\prime}=\frac{\mathrm{AB}}{\mathrm{L}_{1}}
\end{aligned}
$$

Con base en la anterior, se tiene que las relaciones entre las longitudes de estos dos segmentos se obtiene a través de la denominada ley del coseno en el triángulo ABC. Así:

$$
\mathrm{BC}^{2}=\mathrm{AB}^{2}+\mathrm{AC}^{2}-2(\mathrm{AB} \times \mathrm{AC}) \cos 52^{\circ} 16^{\prime}
$$

Después de calcular el valor de $\overline{\mathrm{BC}}$ en términos de $\mathrm{L}$, se requiere calcular el ángulo que se forma entre la diagonal de la puerta abierta y la grieta que hay en la pared $(\theta=\mathrm{BDC})$; para encontrar este ángulo, se hace necesario volver a utilizar la ley de coseno en el triángulo BCD:

$\mathrm{BC}^{2}=\mathrm{BD}^{2}+\mathrm{DC}^{2}-2(\mathrm{BD} \times \mathrm{DC}) \cos \theta$

Como se mencionó antes, en este ejemplo se evoca un fenómeno de movimiento que puede representar una variación del ángulo $\theta$ (cuando se abre o cierra la puerta). Sin embargo, este movimiento puede ignorarse cuando se resuelve la tarea, para reducirse a la presencia de cantidades fijas conocidas, que son constantes ( $v . g$. ángulos de elevación y de depresión) y otras cantidades fijas desconocidas ( $v$. g. largo, ancho y diagonal de la puerta, distancia que hay entre la parte superior izquierda de la puerta y un punto situado llamado $B$, distancia que hay entre el punto situado B y la parte inferior derecha de la puerta, distancia que hay entre el punto situado B y la parte inferior izquierda de la puerta).

Este tipo de tareas ofrece oportunidades para que los estudiantes coordinen una razón trigonométrica con los datos presentados en el enunciado y, a su vez, puedan utilizar algunas expresiones algebraicas para encontrar un resultado. No obstante, tampoco pueden asociarse como una interpretación de las razones trigonométricas desde un punto de vista dinámico, ni a las cantidades que intervienen en ellas (ángulos y los segmentos del triángulo) como variables.

\subsection{Tareas con interpretación dinámica de las razones trigonométricas}

En esta categoría se concentran aquellas tareas que exigen una interpretación de 
fenómenos de cambio y variación. En estas tareas se reconoce la presencia de cantidades fijas y cambiantes y se requiere modelar las relaciones entre ellas. Conforme se mencionó antes, en los textos analizados se encontraron pocas tareas que hicieran referencia a esta tipología. En total se encontraron seis tareas de comparación de estados y ninguna tarea de covariación. Estas tipologías se describen a continuación.

\subsubsection{Tareas por comparación de estados}

Para resolver este tipo de tareas, los estudiantes deben identificar cantidades que varían; su solución atiende al reconocimiento de un cambio promedio descrito en un estado inicial y un estado final. Según las tareas planteadas sobre el estudio de las razones trigonométricas, se distinguen y establecen tres cantidades: inicial, final y de cambio o diferencia entre ellas.

A continuación se presenta, en la Figura 4, un ejemplo de este tipo de tareas.

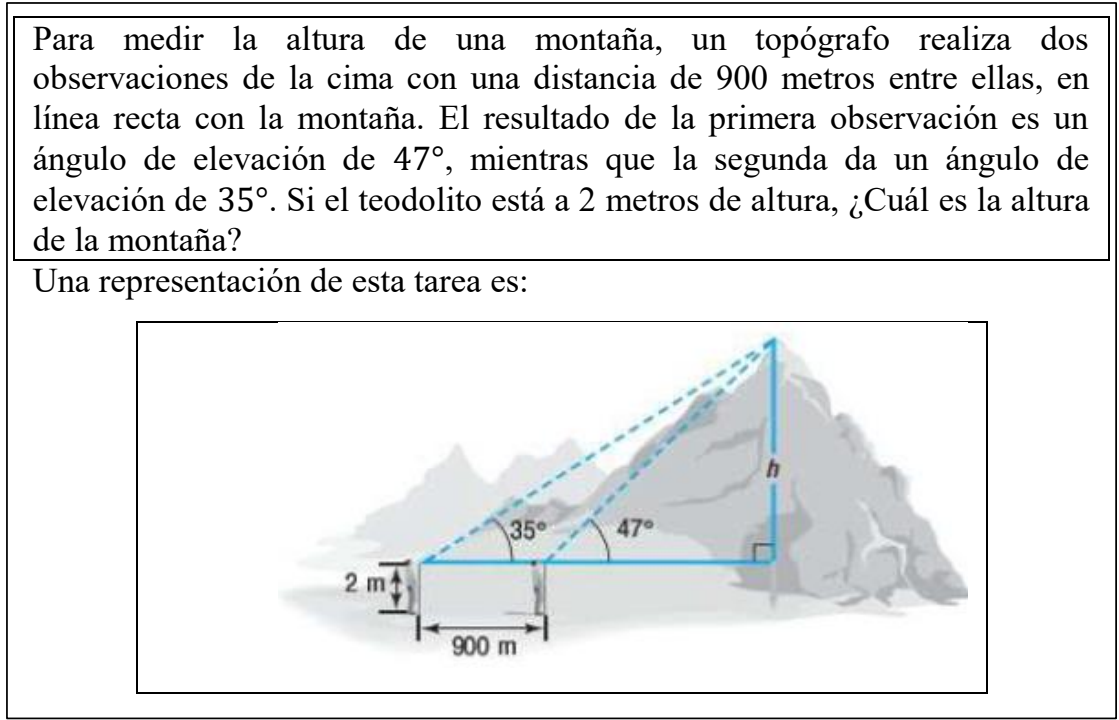

Figura 4 - Representación de la tarea

Fuente: Sullivan (2007, p. 674)

En la gráfica (Figura 4) puede asumirse que $\overline{\mathrm{AC}}$ representa la distancia total que hay entre la montaña y el punto donde quedó parado el topógrafo después de la segunda observación; $\overline{\mathrm{BC}}$ representa la distancia que hay entre el punto de la primera observación y la montaña, $\overline{\mathrm{CD}}$ representa la altura de la montaña (sin incluir la altura del teodolito), $\overline{\mathrm{AD}}$ representa la primera línea visual del topógrafo y la cima de la montaña; $\overline{\mathrm{BD}}$ representa la segunda línea visual del topógrafo y la cima de la montaña.

Este enunciado obedece a las siguientes expresiones algebraicas: 


$$
\begin{aligned}
\tan 35^{\circ} & =\frac{C D}{900+B C} \\
\tan 47^{\circ} & =\frac{C D}{B C}
\end{aligned}
$$

Después de plantear este sistema de ecuaciones lineales $(2 \times 2)$ se obtendría la altura DC $(h)$ a la cual se le debe adicionar la altura del teodolito y obtener la altura de la montaña. En esta tipología de tareas se reitera, una vez más, el cálculo de un valor numérico en particular - altura de la montaña - a pesar de que en este enunciado se utilizan, de forma implícita, algunas palabras que se refieren al cambio y variación en una situación.

En este tipo de tareas la solución atiende al reconocimiento de un cambio en un intervalo que se describe en un estado inicial y un estado final. Conforme se mencionó antes, en las tareas planteadas sobre el estudio de las razones trigonométricas se distinguen y establecen tres cantidades: inicial, final y de cambio o diferencia entre ellas. Esto podría ofrecer una oportunidad para que los estudiantes puedan interpretar la noción de tasa de variación media en el intervalo.

\subsubsection{Tareas de covariación}

En este tipo de tareas, la atención se centra en el cambio de alguna cantidad y el respectivo cambio que genera en otras cantidades variables. Como se evidenció en la Tabla 1, en los libros de texto que se analizaron no se encontró evidencia de la existencia de alguna tarea dentro de esta tipología. Sin embargo, se podría considerar una reorganización de la tarea presentada en la tipología anterior. Por ejemplo, cuestionar por el dominio del ángulo $\operatorname{ADB}(\theta)$ a medida que el observador camina una longitud determinada, alejándose de la montaña, lo cual se puede expresar como una función entre la longitud de un cateto y el ángulo $\theta$.

Se puede adicionar condiciones particulares para preguntar por los mejores ángulos sobre los cuales tomar la medición. Incluso, se puede cuestionar a los estudiantes sobre el número de mediciones que serían necesarias para cumplir el objetivo de la tarea. En la medida de las posibilidades, se esperaría trascender este tipo de tareas estereotipadas para desarrollar tareas auténticas (VILLA-OCHOA; CASTRILLON-YEPES; SÁNCHEZ-CARDONA, 2017), que involucren experimentación, medición y toma de decisiones en la solución de la tarea y contextos en donde las funciones sean útiles para responder a los cuestionamientos que implica. 


\section{Discusión}

Esta revisión a los libros de texto universitarios de precálculo permitió observar una preocupación de sus autores por el uso de fórmulas, con énfasis en un dominio algebraico y procedimental. Esto se evidenció en que la totalidad de las tareas involucraba la aplicación de alguna ecuación de la razón trigonométrica en la que se pedía calcular un valor desconocido. Este hecho sugiere un interés en los textos por el dominio apropiado de símbolos, operaciones y propiedades; pero se desaprovechan oportunidades para promover el estudio de relaciones dinámicas entre las cantidades que intervienen en la tarea.

En consecuencia, las expresiones trigonométricas aparecen como expresiones para hacer cálculos y no para enfatizar en relaciones dinámicas o funcionales entre los ángulos y los lados de los triángulos. Esta característica coincide con los resultados de la investigación de Montiel y Jácome (2014, p. 1213), quienes reconocen, además, que existe un fenómeno de la "pérdida del proceso geométrico en la construcción de lo trigonométrico, donde las razones trigonométricas se convierten en el proceso aritmético de dividir las longitudes de los lados del triángulo". Para los autores, ésto se manifiesta de manera clara en la estructuración del discurso escolar.

En relación con los contextos, las tareas propuestas por los libros de texto evocan contextos realistas (VILLA-OCHOA; CASTRILLON-YEPES; SÁNCHEZ-CARDONA, 2017); es decir, son un conjunto de problemas presentados en enunciados verbales (word problems) que evocan situaciones de potencial uso de las ideas matemáticas, pero poco tienen que ver con la experiencia, necesidades o intereses de los estudiantes en situaciones académicas o cotidianas.

Estas tareas no promueven la experimentación, la toma de decisiones sobre cómo medir y asumir diferentes estrategias para resolver los cuestionamientos de la tarea. Conforme se mencionó, son tareas que ofrecen toda la información numérica y plantean, de manera explícita, la pregunta por una cantidad desconocida. En otras palabras, el enunciado de la tarea proporciona al estudiante toda la información necesaria para resolverla y obtener resultados con base en la repetición, en ocasiones, con la creación de un procedimiento. Esto coincide con el estudio de Montiel y Jácome (2014) quienes, a partir de la investigación de Mesa y Herbst (2011), interpretaron esta situación como la presencia de una norma en el interior de una racionalidad práctica en la que también se involucran los libros de texto.

Si bien en Colombia existe, desde hace más de 20 años, un interés por promover el desarrollo de un pensamiento variacional en los niveles de Educación Básica y Media (seis a 
dieciocho años) (COLOMBIA-MEN, 1998), éste no parece haber permeado a los autores de los libros de texto analizados en este estudio. Se infiere, a partir de este estudio, que las tareas propuestas en los libros de texto pueden ofrecer potenciales oportunidades para una interpretacion de las razones como covariación.

Adicional a las tareas estáticas, se podría equilibrar la presencia de tareas dinámicas que ofrezcan una interpretación de los ángulos y lados de los triángulos como variables; por ejemplo, el cambio del ángulo de observación (elevación) dependiendo del cambio en el crecimiento de un árbol; la distancia de cobertura de una cámara de vigilancia que gira entre determinados ángulos; el cambio en la longitud de una sombra a medida que cambia el ángulo con el sol durante el día. La presencia de este tipo de tareas permitiría extender la presencia de la tipología reportada en este artículo con tareas que se denominaron como tareas de covariación.

Los resultados de este estudio muestran que en los libros de texto que se analizaron existe una dicotomía entre el estudio estático de las razones trigonométricas y el posterior estudio de las funciones trigonométricas. En la historia de las matemáticas, el estudio estático caracterizó la era de las razones y las proporciones. Según Thompson y Carlson (2018), las personas representaban las relaciones entre cantidades de manera geométrica y capturaban la generalidad a través de la similitud; sin embargo, dado que las representaciones eran geométricas, no podían representar el movimiento de forma explícita.

Al respecto, este estudio sugiere que aun cuando las representaciones de las razones trigonométricas sean geométricas, es posible ofrecer oportunidades para otorgarles una interpretación dinámica a través de la covariación de las cantidades que en ellas se involucran. Estas oportunidades son importantes como una manera de generar fundamentos para un estudio posterior de las funciones, pues, tal y como Weber (2005) apunta, la comprensión de una operación trigonométrica como función, exige que los estudiantes reconozcan un proceso en el que puedan usar cualquier ángulo dado para evaluar la función; además, deben desarrollar la capacidad de estimar el resultado de este método y razonar sobre las propiedades del resultado sin necesidad de efectura todos los pasos del proceso.

\section{Conclusiones}

En la primera parte de este artículo se argumentó sobre la necesidad de introducir el estudio de la variación y el cambio en todos los niveles de escolaridad. Ello implica ver el pensamiento variacional como una categoría amplia que recoje los esfuerzos de investigación 
y formación que permitan llevar a cabo esta tarea. En ese sentido, el estudio de la (co)variación puede observarse como un enfoque bajo el cual, diversos objetos de la matemática escolar pueden dotarse de un sentido dinámico (VASCO, 2006).

De acuerdo con estas ideas, este artículo se preocupó por identificar tipos de tareas y las oportunidades que los libros de texto de precálculo ofrecen para un estudio dinámico de las razones trigonométricas. Por lo tanto, a partir de la noción de covariación se buscó dar una respuesta a la pregunta ¿Cuáles tipos de tareas pueden identificarse sobre las razones trigonométricas en libros de texto de precálculo?

Para dar respuesta a la pregunta, la covariación se identificó a través de las oportunidades que se ofrecen para el reconocimiento de la dependencia entre dos o más cantidades variables. Al respecto, este estudio ofrece dos resultados importantes relacionados con el tipo de tareas que puede o no encontrarse en los libros de textos.

El primero de los resultados consiste en una tipologia de tareas. Esta tipologia evidencia que los libros de texto desaprovechan oportunidades para promover interpretaciones de covariación en las tareas sobre razones trigonométricas. Esto se evidenció cuando los procesos de reconocimiento, medición de magnitudes y el análisis y la comprensión de qué y cómo cambian, no se tenían en cuenta en la solución de las tareas.

En palabras de Parada, Conde y Fiallo (2016) se desaprovecha una oportunidad de comprender la variación. Como se argumentó en este artículo, este resultado también puede entenderse como la manifestación de la presencia de una norma en la que todas las tareas deben incluir todos los datos numéricos para que pueda resolverse.

El segundo resultado importante de este artículo muestra que existe la posibilidad de reorganizar las tareas que se aproximen a verdaderas tareas de covariación. Esto sugiere que, aunque las tareas se formulan como enunciados estereotipados de relaciones entre cantidades conocidas y desconocidas, también es posible que los profesores aprovechen los contextos para promover un razonamiento covariacional.

Para Thompson y Carlson (2018), una persona razona covariablemente cuando visualiza los valores de dos cantidades que varían y los ve de forma simultánea; por tanto, las tareas deben promover la coordinación y dependencia de los cambios entre variables y reconocerlas como una componente fundamental de su solución.

Los tipos de tarea que reporta este artículo se convierten en evidencia de que en el contexto colombiano los enunciados verbales rutinarios también parecen ser una herramienta privilegiada para develar un enfoque de lo que Montiel y Jácome (2014, p. 1213) denominaron "la aritmetización trigonometrica". 
Según las tipologías presentes en este artículo, este fenómeno se extiende hacia una omisión del reconocimiento de las cantidades que intervienen en las tareas como cantidades que covarían. Este hecho trae consigo una pérdida de oportunidades para que los estudiantes desarrollen, posteriormente, significados para las funciones trigonométricas como relaciones de cantidades covariables. Según Moore (2014), se requieren esfuerzos para que estudiantes y profesores de trigonometría desarrollen significados para las funciones trigonométricas en los contextos de círculo unitario y triángulo rectángulo; una mayor presencia de la tipología tareas de covariación podría contribuir al alcance de este propósito.

\section{Referencias}

ANTONIO ZAMBRANO, R.; ESCUDERO ÁVILA, D. I.; FLORES MEDRANO, E. Una introducción al concepto de derivada en estudiantes de bachillerato a través del análisis de situaciones de variación. Educación Matemática, Ciudad de México, v. 31, n. 1, p. 258-280, abr. 2019.

ARANDA, C.; CALLEJO, M. L. Construcción de la Función Integral y Razonamiento Covariacional: dos Estudios de Casos. Bolema: Boletim de Educação Matemática, Rio Claro, v. 31, n. 58, p. 777798, ago. 2017.

BARAJAS ARENAS, C.; PARADA RICO, S. E.; MOLINA ZAVALETA, J. G. Análisis de dificultades surgidas al resolver problemas de variación. Educación Matemática, Ciudad de México, v. 30, n. 3, p. 297-323, dez. 2018.

BURITICÁ, B. Álgebra y Trigonometría. Medellín: Universidad de Antioquia, 2012.

CABALLERO-PÉREZ, M.; CANTORAL, R. Una caracterización de los elementos del pensamiento y lenguaje variacional. Acta Latinoamericana de Matemática Educativa, Ciudad de México, v. 26, n. 1, p. 197-1205, 2013.

CABEZAS, C.; MENDOZA, M. R. Manifestaciones emergentes del pensamiento variacional en estudiantes de cálculo inicial. Formación Universitaria, La Serena, v. 9, n. 6, p. 13-26, 2016.

CANTORAL, R. Pensamiento y lenguaje variacional, una mirada socioepistemológica. Acta Latinoamericana de Matemáticas Educativa, Ciudad de México, v.18, n.1, p. 1-9, 2004.

CANTORAL, R.; MONTIEL, G.; REYES-GASPERINI, D. Análisis del discurso matemático escolar en los libros de texto, una mirada desde la teoría socioepistemológica. Avances de Investigación en Educación Matemática, España, n. 8, p. 9-28, 2015.

CARLSON, M. et al. Applying covariational reasoning while modeling dynamic events: A framework and a study. Journal for Research in Mathematics Education, Reston, v. 33, n. 5, p. 352-378, nov. 2002.

COLOMBIA-MEN. Lineamientos curriculares para el área de Matemáticas. Bogotá: Magisterio, 1998.

COLOMBIA-MEN. Estándares Básicos de Competencias Matemáticas. Bogotá: Magisterio, 2006.

FERRARI-ESCOLÁ, M.; MARTÍNEZ-SIERRA, G.; MÉNDEZ-GUEVARA, M. E. M. “Multiply by 
adding": Development of logarithmic-exponential covariational reasoning in high school students. The Journal of Mathematical Behavior, Estados Unidos de América, v. 42, p. 92-108, jun. 2016.

FIALLO-LEAL, J. E.; PARADA-RICO, S. E. Estudio Dinámico del cambio y la variación: Curso de Precálculo Mediado por GeoGebra. Bucaramanga: Ediciones UIS, 2018.

FIALLO-LEAL, J. E.; PARADA-RICO, S. E. Curso de precálculo apoyado en el uso de Geogebra para el desarrollo del pensamiento varacional-Pre calculus course in using supported geogebra for the development of variational thinking. Revista científica, Bogotá, v. 3, n. 20, p. 56-71, oct. 2014.

FLORES, C. D.; GARCÍA-GARCÍA, J. Conexiones Intramatemáticas y Extramatemáticas que se producen al Resolver Problemas de Cálculo en Contexto: un Estudio de Casos en el Nivel Superior. Bolema: Boletim de Educação Matemática, Rio Claro, v. 31, n. 57, p. 158-180, abr. 2017.

MEJÍA, F; ÁLVAREZ, R; FERNÁNDEZ, H. Matematicas previas al cálculo. Medellín: Sello Editorial Universidad de Medellín, 2005

MESA, V.; HERBST, P. Designing representations of trigonometry instruction to study the rationality of community college teaching. ZDM - International Journal on Mathematics Education, Berlin, v. 43, n. 1, p. 41-52, feb. 2011.

MONTIEL, G. E.; BUENDÍA-ÁBALOS, G. Desarrollo del pensamiento funcional-trigonométrico. En: BUENDÍA ÁBALOS, G.; FERRARI-ESCOLÁ, M.; MARTINEZ-SIERRA, G. (Ed.).

Resignificación de funciones para profesores de matemáticas. Ciudad de México: Ediciones Díaz de Santos, 2013. p. 169-205.

MONTIEL, G. E.; JÁCOME, G. Significado Trigonométrico en el Profesor. Bolema: Boletim de Educação Matemática, Rio Claro, v. 28, n. 50, p. 1193-1216, dic. 2014.

MOORE, K. C. Quantitative Reasoning and the Sine Function: The Case of Zac. Journal for Research in Mathematics Education, Reston, v. 45, n. 1, p. 102-138, 2014.

PARADA, S. E.; CONDE, L. A.; FIALLO, J. Mediación Digital e Interdisciplinariedad: una Aproximación al Estudio de la Variación. Bolema: Boletim de Educação Matemática, Rio Claro, v. 30, n. 56, p. 1031-1051, dez. 2016.

PINO, J.; BLANCO, L. Análisis de los problemas de los libros de texto de matemáticas para alumnos de 12 a 14 años de edad de España y de Chile en relación con los contenidos de proporcionalidad.

Publicaciones de la Facultad de Educación y Humanidades del Campus de Melilla, Melilla, n. 38, p. 63-88, 2008.

PINTO, E.; CAÑADAS, M. C. Generalization in fifth grades within a functional approach. PNA, Granada, v. 12, n. 3, p. 173-184, 2018.

RANDAHL, M. Approach to mathematics in textbooks at tertiary level - exploring authors' views about their texts. International Journal of Mathematical Education in Science and Technology, U.K, v. 43, n. 7, p. 881-896, 15 oct. 2012.

RUEDA, J. N.; PARADA-RICO, S. E. Razonamiento covariacional en situaciones de optimización modeladas por Ambientes de Geometría Dinámica. Uni-pluriversidad, Medellín, v. 16, n. 1, p. 51-63, 2016.

SALDANHA, L.; THOMPSON, P. W. Re-thinking covariation from a quantitative perspective: Simultaneous continuous variation. En: BERENSON, S. B.; COULOMBE, W. N. (Ed.). Proceedings of the Annual Meeting of the Psychology of Mathematics Education - North America. Raleigh: North Carolina State University, 1998. v. 1. p. 298-304. 
SELVA, A. C. V.; BORBA, R. E. S. R. El uso de la calculadora en los primeros grados de educación básica. Medellín: Sello Editorial Universidad de Medellín, 2014.

SILVA, M. A. et al. Brazilian High School Mathematics Textbooks and the Constitution of the Good Student Citizen. Acta Scientiae, Canoas, v. 20, n. 6, p. 1071-1081, dez. 2018.

SON, J.; DILETTI, J. What Can We Learn from Textbook Analysis? En: SON, J.-W.; WATANABE, T.; LO, J.-J. (Eds.). What Matters? Research Trends in International Comparative Studies in Mathematics Education. Cham: Springer International Publishing, 2017. p. 3-32.

STEPHENS, A. C. et al. A Learning Progression for Elementary Students' Functional Thinking. Mathematical Thinking and Learning, Estados Unidos de América, v. 19, n. 3, p. 143-166, 3 jul. 2017.

SULLIVAN, M. Álgebra y Trigonometría. México: Pearson, 2007

TAVERA, F.; VILLA-OCHOA, J. A. La variación en algunos textos universitarios. El caso de las relaciones trigonométricas. Acta Latinoamericana de Matemática Educativa, Ciudad de México, v. 28, p. 394-400, 2015.

TALL, D. O. Dynamic mathematics and the blending of knowledge structures in the calculus. ZDM Mathematics Education, Berlin, v. 41, n. 4, p. 481-492, 8 ago. 2009.

THOMPSON, P. W.; CARLSON, M. Variation, covariation and functions: Foundational ways of mathematical thinking. En: CAI, J. (Ed.). Compendium for Research in Mathematics Education. Reston: NCTM, 2018. p. 421-456.

THOMPSON, P. W. Images of rate and operational understanding of the fundamental theorem of calculus. Educational Studies in Mathematics, Dordrecht, v. 26, n. 2-3, p. 229-274, mar. 1994.

VASCO, C. E. Pensamiento variacional, la modelación y las nuevas tecnologías. En: VASCO, C. E. (Ed.). Didáctica de las matemáticas: artículos selectos. Bogotá: Universidad Pedagógica Nacional, 2006. p. 134-148.

VILLA-OCHOA, J. A. Razonamiento covariacional en el estudio de funciones cuadráticas. TED: Tecné, Episteme y Didaxis, Bogotá, n. 31, p. 9-25, mar. 2012.

VILLA-OCHOA, J. A.; GONZÁLEZ-GÓMEZ, D.; CARMONA-MESA, J. A. Modelación y Tecnología en el Estudio de la Tasa de Variación Instantánea en Matemáticas. Formación universitaria, La Serena, v. 11, n. 2, p. 25-34, abr. 2018.

VILLA-OCHOA, J. A.; CASTRILLÓN-YEPES, A.; SÁNCHEZ-CARDONA, J. Tipos de tareas de modelación para la clase de matemáticas. Espaço Plural, Cascavel, v. 18, n. 36, p. 219-251, 2017.

WEBER, K. Students' understanding of trigonometric functions. Mathematics Education Research Journal, Australia, v. 17, n. 3, p. 91-112, oct. 2005. 